\title{
Pilot Assignment Based on Graph Coloring and Location Information in Multicell Multiuser Massive MIMO Systems
}

\author{
Canyun Xiong, Shiyong Chen, Liang Li, and Yucheng Wu \\ School of Microelectronics and Communication Engineering, Chongqing University, Chongqing, China \\ Correspondence should be addressed to Yucheng Wu; wuyucheng@cqu.edu.cn
}

Received 12 March 2021; Revised 16 June 2021; Accepted 2 July 2021; Published 15 July 2021

Academic Editor: Chi-Hua Chen

Copyright (C) 2021 Canyun Xiong et al. This is an open access article distributed under the Creative Commons Attribution License, which permits unrestricted use, distribution, and reproduction in any medium, provided the original work is properly cited.

\begin{abstract}
A massive multiple-input multiple-output (MIMO) system uses a large number of antennas in the base station (BS) to serve multiple users, which significantly improves the capacity of the system. However, in time division duplex (TDD) mode, the pilot contamination (PC) is inevitable due to the multiplexing of pilots. This paper proposed a pilot assignment based on graph coloring and location information (GC-LI) to improve the performance of users. Specifically, based on graph coloring, the proposed GC-LI algorithm combines location information like the angle of arrival (AoA), distance, and correlation to construct an interference graph. Then, we calculate the interference between any two users and use the postprocessing discrete Fourier transform (DFT) filtering process to effectively distinguish the users with nonoverlapping AoAs. Finally, according to the interference graph, the GC-LI algorithm is proposed to mitigate the intercell interference (ICI) between users with the same pilot by assigning different pilots to connected users with high ICI metrics based on some regulation. Simulation results show that the GC-LI algorithm is suitable for various types of cells. In addition, compared with the existing pilot assignment algorithms based on graph coloring, users' average signal-to-interference-plus-noise ratio (SINR) and uplink achievable sum rate (ASR) are significantly improved.
\end{abstract}

\section{Introduction}

The $5 \mathrm{G}$ network is regarded as the foundation of the Internet of Things (IoT), Internet of Vehicles (IoV), and other things in the future $[1,2]$. Massive multiple-input multiple-output (MIMO) technology significantly improves the system capacity and spectrum utilization, which has become a key technology and a necessary component of 5G $[3,4]$. However, in time division duplex (TDD) mode, limited by the coherent time and bandwidth of the actual wireless channel, the number of orthogonal pilots is limited [5].

As the number of antennas increases, the user's channels are orthogonal gradually, so the small-scale fading and irrelevant thermal noise disappear by degrees [6]. However, users who multiplex the same pilot will cause the threshold effect, which will affect the channel estimation accuracy and make the system performance saturated. The phenomenon that the users' uplink and downlink are interfered with due to multiplexing of the pilot sequences is called pilot contamination (PC) [7]. For high-capacity dense scenes, the PC problem will be more serious. Therefore, the PC suppression technology has become a research focus, especially in 5G scenarios; it has become the essential of massive MIMO technology $[8,9]$.

Typically, there are three ways to suppress PC in a massive MIMO system: efficient channel estimation algorithms, appropriate precoding methods, and better pilot assignment strategies. The channel estimation algorithms enhance the system performance by improving the accuracy of channel state information (CSI) estimation [10-12]. Some researchers use the estimated CSI to design a suitable precoding structure of downlink data. When BS receives the data, the channel interference on the transmitted data can be eliminated by decoding $[13,14]$. The pilot assignment strategies assign the best pilots by measuring the interference strength to mitigate ICI among users who reuse pilots [15]. From the above analysis, we can see that the method based on pilot assignment is the premise of the other two methods, so this paper focuses on the pilot assignment strategy to suppress the PC problem.

Several approaches have been proposed to mitigate the pilot contamination effect in multicell massive MIMO 
systems. Reference [16] modifies the position of pilots in each cell of the frame structure to ensure that the period of pilots transmitted by each cell nonoverlapping. Reference [17] further combines with pilot power control to improve the uplink and downlink achievable rate of users. However, this method influences the pilot information of the received data. The algorithm proposed in [18] is based on the idea of reducing the pilot transmitted power of interfering users, which eliminates the interference by utilizing the different signal power arriving at the base station (BS) of the target user and the interfering users. In [19], a joint pilot design and uplink power allocation algorithm is proposed to optimize max-min spectral efficiency directly. To facilitate optimization, a pilot design where the pilot signals are treated as continuous variables is proposed. The algorithm can obtain significant gains with polynomial complexity. However, the complexity of the algorithm is still very high. Reference [20] proposes a multiobjective optimization method for pilot design in a multicell massive MIMO system. The proposed approach simultaneously minimizes the total channel estimation errors at the BSs and the total pilot power consumed by all users. However, pilots for users within the same cell are not orthogonal in the pilot design scheme. Thus, it increases intracell interference compared with conventional orthogonal pilot design. A pilot assignment method based on the performance degradation of users is proposed in [21], which divides users into central users and edge users by certain criteria to improve the SINR of edge users by using additional orthogonal pilots. In [22], a pilot assignment method based on user grouping is proposed. For the central users, pilots are randomly assigned. As for the edge users, they are first sorted according to their direct-view path interference metrics, and then, the user with the largest interference metric is assigned pilot firstly. However, this method reduces the pilot multiplexing gain and increases the overhead. In $[23,24]$, the pilot assignment methods based on graph coloring are proposed. Both take the large-scale fading coefficient as a metric to establish an interference graph for all users in multicell. Then, the pilots are dynamically allocated to improve the overall performance of the system. However, the interference graph established by this method is not accurate enough. In [25], a multilayer fully connected deep neural network model is constructed. The large-scale fading coefficient is used to balance the pilot transmitted power of each user to reduce the channel estimation error. A neural network model is established in [26]. The user's location is used to establish a spatial location model, which takes the entire system as input, and quickly obtains the pilot assignment scheme for all users. However, the training model based on deep learning is more complex.

Given the requirement of high capacity and high transmission rate in the actual 5G scenario [27-29], the cell's radius covered by each BS is becoming smaller. The effects of traditional pilot assignment algorithms are not ideal. Therefore, a pilot assignment scheme based on graph coloring and location information (GC-LI) is proposed in this paper. The main contributions of this study are summarized as follows:

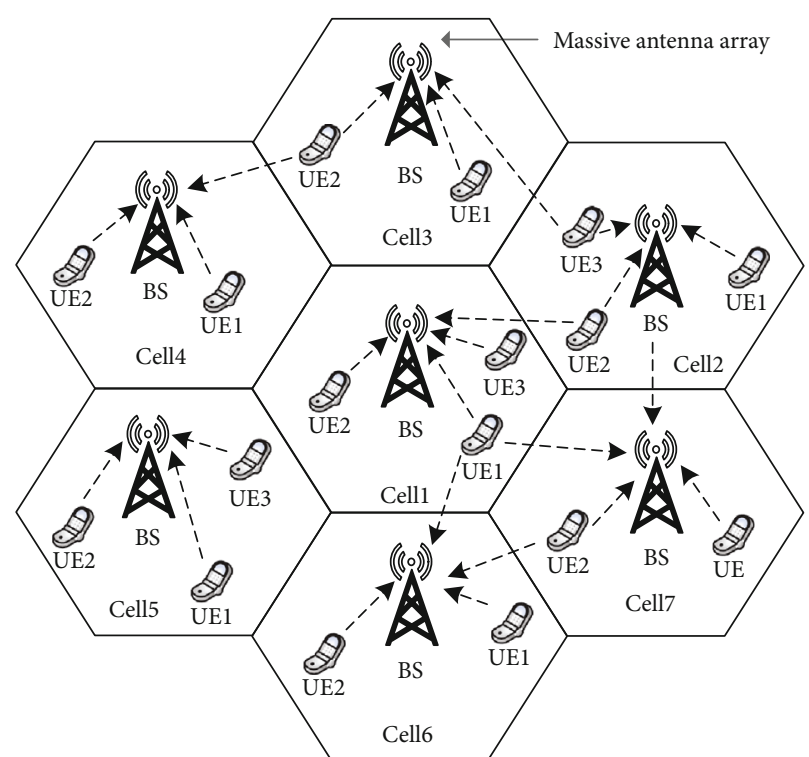

FIGURE 1: Schematic diagram of the multicell multiuser massive MIMO scenario.

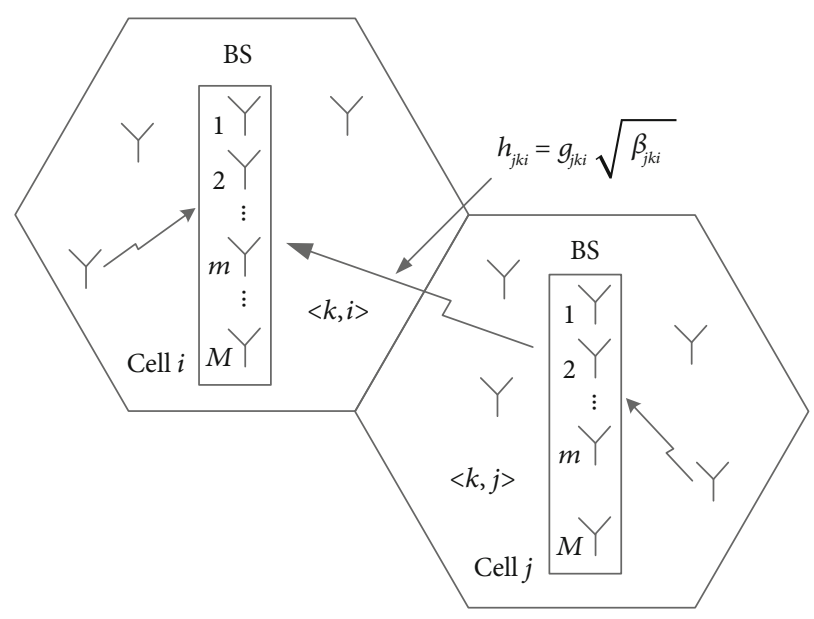

FIgURE 2: Signal transmission model of multicell multiuser.

(1) The interference model is constructed by using the idea of graph coloring. The location information is further combined in the construction of the interference graph, which considers the influence of the large-scale fading coefficient caused by the distance and the angle of arrival (AoA) of users

(2) The postprocessing discrete Fourier transform (DFT) filtering process is used to filter out the pilot interference of users with nonoverlapping AoAs. The potential intercell interference (ICI) strength between any two users in different cells is measured, and an interference graph including all cell users is constructed

(3) For the multicell multiuser massive MIMO system, a pilot assignment algorithm GC-LI is proposed to reduce the ICI in the system. We assign suitable pilots 
to users based on some criteria to ensure connected users with a large ICI strength metric which will not be assigned the same pilot sequence. Simulation results show that the proposed scheme can improve the average signal-to-interference-plus-noise ratio (SINR) and achievable sum rate (ASR) of cells

The remainder of this paper is introduced as follows. Section 2 shows the system model and describes the cause of PC. A pilot assignment algorithm is proposed in Section 3. Section 4 presents the simulation results. Finally, the conclusion is summarized in Section 5.

\section{System Model}

This section introduces the multicell multiuser massive MIMO system and its channel model. Then, a detailed derivation and analysis of the primary cause of PC are carried out. Finally, the principle of DFT to eliminate PC is explained in detail.

2.1. System and Signal Model. As shown in Figure 1, a multicell multiuser massive MIMO system is considered, which consists of $L$ hexagonal cells. Each cell consists of $M$ antennas and $K(M \gg K)$ users with a single antenna. The dashed arrow indicates the data or pilot received by the BS from users. We can see that the BS will also receive pilots from users in neighboring cells. When the pilots used by the neighboring cells are the same as the target cell, it will cause interference.

Figure 2 describes the multicell multiuser signal transmission model through two cells to further analyze the cells' interaction. When users in the cells transmit pilots in the cochannel simultaneously, the channel propagation matrix $\mathbf{h}_{j k i}$ from user $k$ in cell $j$ to the BS in cell $i$ can be expressed as

$$
\mathbf{h}_{j k i}=\mathbf{g}_{j k i} \sqrt{\beta_{j k i}}, \quad i, j \in\{1, \cdots, L\} ; k \in\{1, \cdots, K\}
$$

where $\mathbf{g}_{j k i} \in \mathbb{C}^{M \times 1}$ denotes the small-scale fading coefficient that obeys a cyclic complex Gaussian distribution with the mean value of 0 and the variance of $\mathbf{I}_{M}$, namely, $\mathbf{g}_{j k i} \sim C$ $N\left(0, \mathbf{I}_{M}\right) . \beta_{j k i}$ denotes the large-scale fading coefficient that can be expressed as

$$
\beta_{j k i}={\frac{z_{j k i}}{r_{j k i} / R}}^{\alpha}
$$

where $z_{j k i}$ indicates the shadow fading that obeys the lognormal distribution, that is, $10 \lg \left(z_{j k i}\right)$ obeys the Gaussian distribution with the mean value of 0 , and the standard deviation of $\delta_{\text {shadow }}$, whereby $\delta_{\text {shadow }}$ is the shadow fading factor. $r_{j k i}$ indicates the direct viewing distance from user $k$ in cell $j$ to the BS in cell $i$. $R$ indicates the cell radius, $\alpha$ indicates the path loss factor, and $z_{j k i}, r_{j k i}, \alpha$ are considered independent of each other.

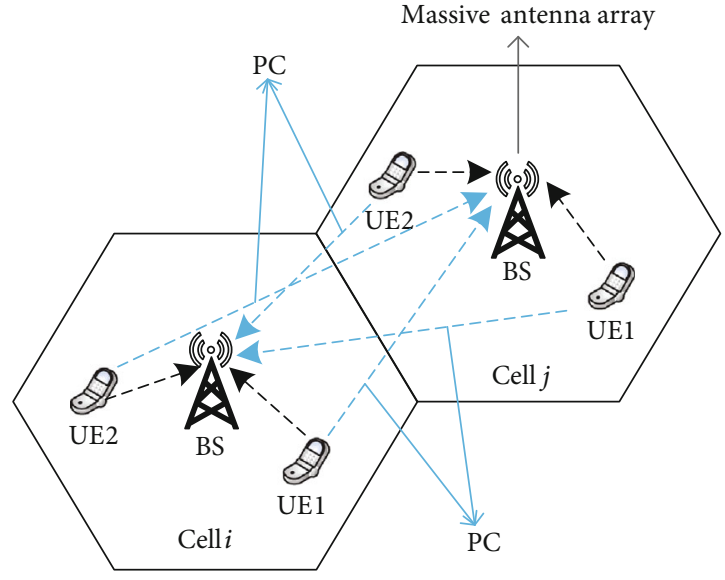

FIgure 3: The illustration of uplink PC.

To simplify the analysis, it is generally believed that the antenna arrays used in a massive MIMO system are closely arranged. Therefore, we considered the large-scale fading coefficient of the signal sent by the user to each antenna of the BS is equal, and the large-scale fading coefficient of different users arriving at the BS is independent with each other. The channel transmission matrix from all $K$ users in cell $j$ to the BS of cell $i$ can be expressed as

$$
\mathbf{H}_{j i}=\left[\mathbf{h}_{j 1 i}, \mathbf{h}_{j 2 i}, \cdots, \mathbf{h}_{j k i} \cdots, \mathbf{h}_{j K i}\right]=\mathbf{G}_{j i}\left(\mathbf{D}_{j i}\right)^{1 / 2},
$$

where $\mathbf{G}_{j i}=\left[\mathbf{g}_{j 1 i}, \mathbf{g}_{j 2 i}, \cdots, \mathbf{g}_{j k i} \cdots, \mathbf{g}_{j K i}\right]$ is a matrix, it is constructed by small-scale fading coefficients of all $K$ users in cell $j$, and $\mathbf{D}_{j i}=\operatorname{diag}\left(\beta_{j 1 i}, \beta_{j 2 i}, \cdots, \beta_{j k i} \cdots, \beta_{j K i}\right)$ is a $K \times K$ diagonal matrix which is composed of the large-scale fading coefficient of all $K$ users.

The channel autocorrelation among all users in cell $j$ to cell $i$ BS is analyzed as

$$
\lim _{M \longrightarrow \infty}\left(\frac{\mathbf{H}_{j i}^{H} \mathbf{H}_{j i}}{M}\right)=\lim _{M \longrightarrow \infty}\left(\mathbf{D}_{j i}^{1 / 2}\right)^{H} \frac{\mathbf{G}_{j i}^{H} \mathbf{G}_{j i}}{M}\left(\mathbf{D}_{j i}^{1 / 2}\right)=\mathbf{D}_{j i} \delta_{j i},
$$

where $\delta_{j i}=1$ when $j=i$ else $\delta_{j i}=0$ when $j \neq i$. When the number of antennas approaches infinity, the small-scale fading coefficient $\mathbf{G}_{\mathbf{j i}}$ of different users to the BS will tend to 0, only affected by $\mathbf{D}_{j i}$.

2.2. Generation and Suppression of PC. In TDD mode, the uplink and downlink data transmission between users and the BSs is based on channel reciprocity. The channel coherence time can be divided into four stages: uplink data transmission, uplink pilot transmission, signal processing in BS, and downlink data transmission. The fundamental reason for PC lies in reusing the same pilot in the uplink, which involves the first three stages. 
2.2.1. Uplink Data Transmission. Taking the model in Figure 2 as an example, according to $\mathbf{h}_{j k i}$ obtained in formula (1), the data $\mathbf{Y}_{i}^{u}$ received by the BS in cell $i$ is given by

$$
\mathbf{Y}_{i}^{u}=\sqrt{P_{u}} \sum_{j=1}^{L} \sum_{k=1}^{K} \mathbf{h}_{j k i} x_{j k}^{u}+\mathbf{N}_{i}^{u}
$$

where $P_{u}$ is the average uplink transmitted power of data, and $x_{j k}^{u}$ denotes the data transmitted by user $k$ in cell $j$, which satisfied $E\left\{\left|x_{j k}^{u}\right|^{2}\right\}=1 . \mathbf{N}_{i}^{u} \in \mathbb{C}^{M \times 1}$ denotes the additive white Gaussian noise (AWGN) satisfying

$$
E\left\{\mathbf{N}_{i}^{u}\left(\mathbf{N}_{i}^{u}\right)^{H}\right\}=\left(\delta_{n}^{u}\right)^{2} \mathbf{I}_{M}
$$

where $\left(\mathbf{N}_{i}^{u}\right)^{H}$ represents the Hermitian transposition of noise vector $\left(\mathbf{N}_{i}^{u}\right)$.

2.2.2. Uplink Pilot Transmission. This paper is based on the multiplexing pilot assignment, so the cells use the same set of pilot sequences, which are assigned to users in each cell. $\boldsymbol{\Phi}=\left(\varphi_{1}, \varphi_{2}, \cdots, \varphi_{k}, \cdots, \varphi_{K}\right)^{T}$ represents the $k \times \tau$ dimensional pilot sequence matrix allocated to all $K$ users in cell $i$. $\tau$ is the length of the pilot sequence, which satisfies the orthogonality between pilots, namely, $\boldsymbol{\Phi} \boldsymbol{\Phi}^{H}=I_{K} . I_{K}$ is a $K \times K$ dimension unit matrix. Finally, the pilot $\mathbf{Y}_{i}^{p}$ received by the BS in cell $i$ can be expressed as

$$
\mathbf{Y}_{i}^{p}=\sqrt{P_{p}} \sum_{j=1}^{L} \sum_{k=1}^{K} \mathbf{h}_{j k i} \varphi_{k}+\mathbf{N}_{i}^{p}
$$

where $P_{p}$ is the average uplink transmitted power of pilots, $\varphi_{k}$ is the pilot sequences assigned by the $k^{\text {th }}$ user in each cell, and $\mathbf{N}_{i}^{p}$ represents the $M \times \tau$ additive white Gaussian noise (AWGN) matrix with independent and identically distributed zero-mean and variance $\left(\delta_{n}^{p}\right)^{2}$.

2.2.3. Signal Processing in BS. The BS performs channel estimation on the received pilots to obtain the estimated value. It then performs matched filtering on the received data and finally obtains the users' original data. In this study, the least square (LS) algorithm is used to estimate the channel. By multiplying the received pilots with the conjugate transposed $\boldsymbol{\Phi}^{H}$ of the known pilot sequences, the channel estimation value $\widehat{\mathbf{H}}_{i i}^{L S}$ of all $K$ users in cell $i$ is obtained as

$$
\widehat{\mathbf{H}}_{i i}^{L S}=\frac{1}{\sqrt{P_{p}}} \mathbf{Y}_{i}^{p} \boldsymbol{\Phi}^{H}=\mathbf{H}_{i i}+\sum_{j=1, j \neq i}^{L} \mathbf{H}_{j i}+\frac{1}{\sqrt{P_{p}}} \mathbf{N}_{i}^{p} \boldsymbol{\Phi}^{H} .
$$

Therefore, for user $t$ in the target cell $i$, the estimated channel transmission matrix is as

$$
\widehat{\mathbf{h}}_{i t i}^{L S}=\mathbf{h}_{i t i}+\sum_{j=1, j \neq i}^{L} \mathbf{h}_{j t i}+n .
$$

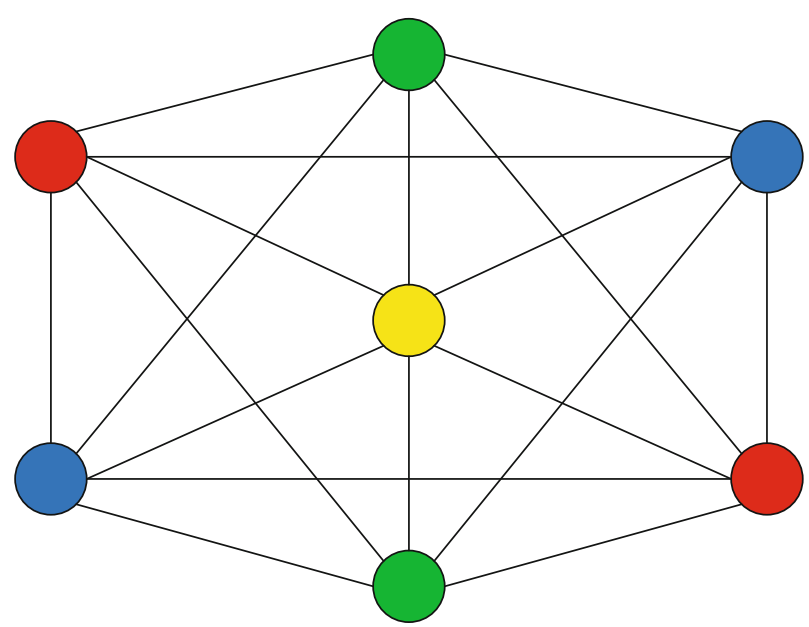

FIgURE 4: Schematic diagram of vertex coloring diagram of the graph.

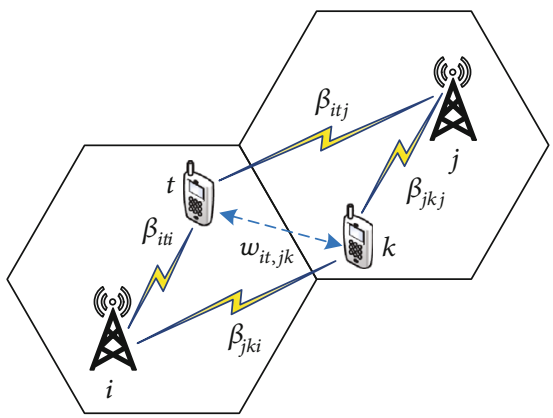

FIGURE 5: Schematic diagram of interference measurement between users.

From formula (9), we can see that the channel estimation value $\widehat{\mathbf{h}}_{i t i}^{L S}$ of user $t$ in cell $i$ includes three parts. The first part is the theoretical channel transmission matrix from user $t$ to the $\mathrm{BS}$ in cell $i$. The second part is the channel transmission matrix from other users using the same pilot sequence to the BS in cell $i$. This part is the PC caused by users who reuse the same pilot in other cells, as shown in Figure 3. The last part $n$ is noise.

2.2.4. Postprocessing DFT Filtering Process. The multiplexing of the pilots in the target cell by the adjacent cells will cause the channel estimation to be distorted. According to the actual application environment, we consider the narrowband multipath signal model.

$$
\mathbf{h}_{i t i}=\frac{1}{\sqrt{N}} \sum_{n=1}^{N} \alpha\left(\theta_{t n}\right) \beta_{t n}
$$

where $N$ is the number of multipaths from user $t$ of target cell $i$ to BS of cell $i, \alpha\left(\theta_{t n}\right)$ is the direction vector of the $n^{\text {th }}$ path of user $t$ to the BS, and $\beta_{t n}$ is the large-scale fading coefficient of the $n^{\text {th }}$ path, including path loss and shadow fading. $\alpha\left(\theta_{t n}\right)$ can be expressed as

$$
\alpha\left(\theta_{t n}\right)=\left[1 e^{-j 2 \pi(D / \lambda) \cos \left(\theta_{t n}\right)} \cdots e^{-j 2 \pi((M-1) D / \lambda) \cos \left(\theta_{t n}\right)}\right]^{T},
$$


where $\lambda$ is the wavelength and $D \leq \lambda / 2$ is the spacing between the antennas of the BS. $\theta \in[0, \pi]$ is the AOA of the arrival signal (owing to $\cos (\theta)=\cos (-\theta)$, only $\theta \in[0, \pi]$ is considered, and $\theta \in[-\pi, 0]$ can be replaced).

Based on Theorem 1 in [30], for the $n^{\text {th }}$ path of user $t$, $\alpha\left(\theta_{t n}\right)$ can be expressed as a monophonic signal whose frequency information is $f_{t n}=\cos \left(\theta_{t n}\right) * D / \lambda$. With the increase of the number of antennas, the Fourier transform of $\alpha\left(\theta_{t n}\right)$ tends to the $\delta$ function after normalization

$$
F_{W}\left(\alpha\left(\theta_{t n}\right)\right)= \begin{cases}F_{W}(f), & f=f_{t n} \\ 0, & \text { else. }\end{cases}
$$

where $F_{W}(\cdot)$ stands for the $W$ point DFT, and $W \geq M$. $F_{W}(f)$ is the DFT value of $f_{t n}$.

Therefore, considering the $N$ paths of user $t$, an angle interval $\left[\theta_{t}^{\min }, \theta_{t}^{\max }\right]$ is defined, which satisfies $\theta_{t}^{\min } \leq \theta_{t n} \leq$ $\theta_{t}^{\max }, n=1,2, \cdots, N$. The position of DFT points in the frequency domain corresponding to the angle is given by

$$
w(\theta)= \begin{cases}W-W \frac{D}{\lambda} \cos (\theta), & \theta \in\left[0, \frac{\pi}{2}\right], \\ -W \frac{D}{\lambda} \cos (\theta), & \theta \in\left[\frac{\pi}{2}, \pi\right] .\end{cases}
$$

The frequency-domain position interval $I_{F}\left(w_{\min }, w_{\max }\right)$ corresponding to the angle interval $\left[\theta_{t}^{\min }, \theta_{t}^{\max }\right]$ is given by $I_{F}\left(w_{\min }, w_{\max }\right)= \begin{cases}{\left[0, w_{\max }\right] \cup\left[w_{\min }, W\right],} & 0 \leq \theta_{t}^{\min }<\frac{\pi}{2}<\theta_{t}^{\max } \leq \pi, \\ {\left[w_{\min }, w_{\max }\right],} & \text { else, }\end{cases}$

where

$$
\left\{\begin{array}{l}
w_{\min }=w\left(\theta_{t}^{\min }\right), \\
w_{\max }=w\left(\theta_{t}^{\max }\right) .
\end{array}\right.
$$

Therefore, the normalized Fourier transform of the direction vector of all paths from user $t$ to the BS in cell $i$ can be expressed as

$$
F_{W}(\alpha(\theta))= \begin{cases}F_{W}(w), & w \in I_{F}\left(w_{\min }, w_{\max }\right), \\ 0, & w \notin I_{F}\left(w_{\min }, w_{\max }\right) .\end{cases}
$$

According to formula (16), when the AOAs of the multiplexing user and the target user are nonoverlapping, the interference from the multiplexing user can be eliminated.

When the DFT value outside the interval is set to zero, the DFT inverse transformation is carried out to get $f_{W}$

$$
f_{W}=\operatorname{IFFT}\left(F_{W}(\alpha(\theta))\right)=\left[f_{1} f_{2} \cdots f_{W}\right]^{T} .
$$

The channel transfer matrix of user $t$ in the target cell $i$ is

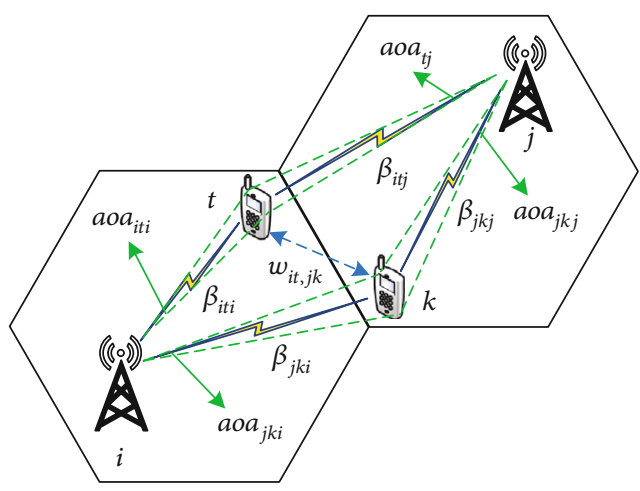

FIGURE 6: Schematic diagram of interference measurement between users with combining location information.

obtained by taking $M$ points

$$
\widehat{\mathbf{h}}_{i t i}=\left[f_{1} f_{2} \cdots f_{M}\right]^{T} .
$$

It can be concluded that the uplink asymptotic $\operatorname{SINR}_{i t}$ of user $t$ in cell $i$ is as

$$
\operatorname{SINR}_{i t}=\frac{\left|\mathbf{h} \wedge{ }_{i t i}^{H} \mathbf{h}_{i t i}\right|^{2}}{\sum_{j=1, j \neq i}^{L}\left|\mathbf{A}_{j t i} \mathbf{h} \wedge_{j t i}^{H} \mathbf{h}_{j t i}\right|^{2}+n_{i}},
$$

where $\mathbf{A}_{j t i}$ is the detection vector of all users in cell $j$ received by user $t$ in cell $i . n_{i}$ is the power of uncorrelated interference and noise. The uplink achievable rate $C_{i t}$ is given by

$$
C_{i t}=\left(1-\mu_{s}\right) E\left\{\log _{2}\left(1+\operatorname{SINR}_{i t}\right)\right\}
$$

where $\mu_{s}=(\tau / K) \mu_{0}$ is the spectrum efficiency loss caused by pilot overhead, and $\mu_{0}$ is the total multiplexing pilot cost coefficient. From formula (19) and (20), we can see that the average SINR and ASR of the system can be improved by suppressing PC.

\section{A Pilot Assignment Based on Graph Coloring and Location Information}

Graph coloring means that all vertices connected by edges have different colors. The pilot assignment in a multicell multiuser massive MIMO system can also be understood as a vertex coloring problem. Users are equivalent to vertices, pilots are equivalent to colors, and the interference value among users is equivalent to edges' weight.

Figure 4 is a vertex coloring diagram. Generally, a graph can be represented by a $G=[V, E], V$ is the set of all vertices, and $E$ is the set of all edges in the graph. In a multicell multiuser system, the pilot assigned randomly in each cell will lead to the pilot multiplexed by multiple users at the cell edge, which will greatly degrade the performance of edge users. Therefore, the interference among all users in multicell should be considered simultaneously. 


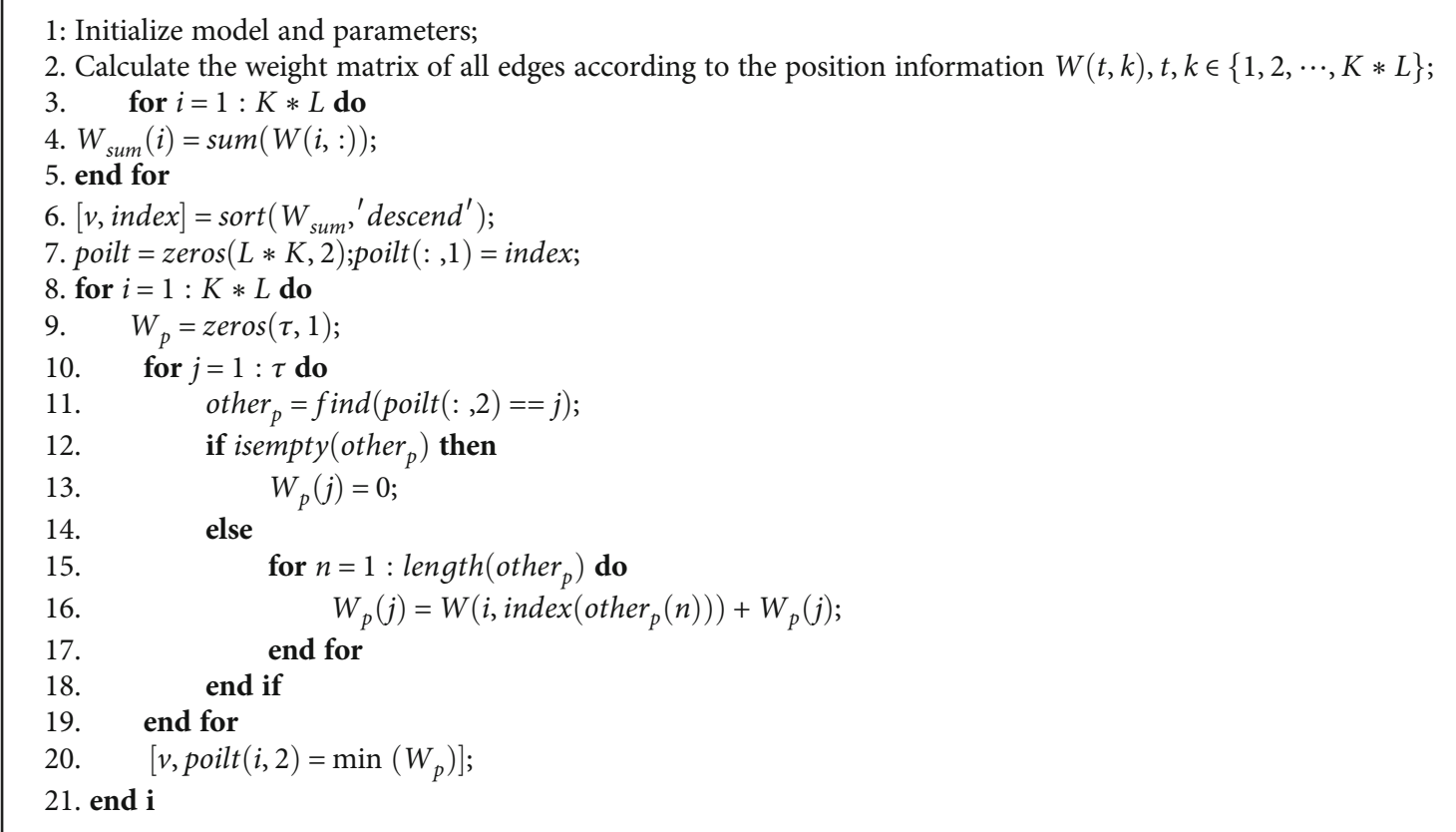

Algorithm 1: Proposed GC-LI algorithm

3.1. Construction of Interference Graph. Combined with the overview of the graph coloring, a model is constructed by using the graph coloring method to realize the pilot assignment in multicell multiuser massive MIMO systems. Firstly, the interference value among users is taken as the weight to construct the interference graph.

The interference graph is constructed according to the large-scale fading coefficients $\beta_{j k i}$, which denotes the channel strength between the users and the BSs. As shown in Figure 5, the interference value $w_{<i t, j k>}$ between user $t$ in cell $i$ and user $k$ in cell $j$ can be expressed as

$$
w_{<i t, j k>}=\frac{\beta_{j k i}^{2}}{\beta_{i t i}^{2}}+\frac{\beta_{i t j}^{2}}{\beta_{j k j}^{2}}, \quad i, j \in L ; k, t \in K,
$$

where $\beta_{j k i}$ denotes the large-scale fading coefficient from user $k$ in cell $j$ to the BS in cell $i, \beta_{i t i}$ denotes the large-scale fading coefficient from user $t$ in cell $i$ to the BS in cell $i, \beta_{i t j}$ denotes the large-scale fading coefficient from user $t$ in cell $i$ to the BS in cell $j$, and $\beta_{j k j}$ denotes the large-scale fading coefficient from user $k$ in cell $j$ to the BS in cell $j$.

The proposed GC-LI algorithm in this paper further combines the location information when constructing the interference graph. It not only considers the large-scale fading coefficient caused by users' position but also considers their AoAs. The ICI of users with nonoverlapping AoAs is eliminated by using the postprocessing DFT filtering process. Therefore, when the interference graph is established, the weight of the edge with nonoverlapping AoAs between users is set to 0 . As shown in Figure 6, it noted that user $t$ in cell $i$ and user $k$ in cell $j$ have different angles to reach the BSs. Four situations should be considered simultaneously to construct the interference graph more accurately.

(1) The AoAs of users arriving at the BSs are nonoverlapping

(2) The AoAs of users arriving at the BS in cell $i$ are nonoverlapping, and arriving at the BS in cell $j$ are overlapping

(3) The AoAs of users arriving at the BS in cell $i$ are overlapping, and arriving at the BS in cell $i$ are nonoverlapping

(4) The AoAs of users arriving at the BSs are overlapping

For the four cases, we consider the interference of the nonoverlapping part of AoAs to be 0 . Therefore, the interference metric $w_{<i t, j k>}$ between user $t$ in cell $i$ and user $k$ in cell $j$ can be expressed as

$$
w_{<i t, j k>}= \begin{cases}0, & \operatorname{aoa}_{i t i} \cap \mathrm{aoa}_{j k i} \in \varnothing, \mathrm{aoa}_{i t j} \cap \mathrm{aoa}_{j k j} \in \varnothing, \\ \frac{\beta_{i t j}^{2}}{\beta_{j k j}^{2}} & \text { ooa }_{i t i} \cap \mathrm{aoa}_{j k i} \in \varnothing, \mathrm{aoa}_{i t j} \cap \mathrm{aoa}_{j k j} \notin \varnothing, \\ \frac{\beta_{j k i}^{2}}{\beta_{i t i}^{2}} & \text { aoa }_{i t i} \cap \mathrm{aoa}_{j k i} \notin \varnothing, \mathrm{aoa}_{i t j} \cap \mathrm{aoa}_{j k j} \in \varnothing, \\ \frac{\beta_{j k i}^{2}}{\beta_{i t i}^{2}}+\frac{\beta_{i t j}^{2}}{\beta_{j k j}^{2}}, & \text { else, }\end{cases}
$$


where aoa ${ }_{j k i}$ represents the AoA range from user $k$ in cell $j$ to the BS in cell $i$, aoa $_{i t i}$ represents the AoA range from user $t$ in cell $i$ to the BS in cell $i$, aoa ${ }_{i t j}$ represents the AoA range from user $t$ in cell $i$ to the BS in cell $j$, and aoa ${ }_{j k j}$ represents the AoA range from user $k$ in cell $j$ to the BS in cell $j$. If the intersection of the AoA ranges of the users is empty, the AoAs are nonoverlapping.

Therefore, in a multicell multiuser massive MIMO system, the cell is numbered $\{1,2, \cdots, L\}$, and cell users are numbered $\{1,2, \cdots, L, \cdots, 2 L, \cdots, K * L\}$. The weight matrix $W$ of the edge set $E$ in the interference graph $G=[V, E]$ can be expressed as

$$
W_{t, k}=w_{\text {vec } 1, \text { vec } 2 k}, \quad k, t \in\{1,2, \cdots, K * L\},
$$

where vec $1 t$, vec $2 k$ represents the cell number of the user $t$ and user $k$, respectively.

3.2. Proposed GC-LI Algorithm. For a multicell multiuser massive MIMO system, the pilots are assigned for vertices after constructing the interference graph. After calculating the degree of each vertex, the pilots are assigned to users with higher vertex degrees to balance the performance of the system. The proposed GC-LI algorithm is shown in Algorithm 1, which is briefly explained and analyzed.

In Steps 1-2, according to the related system parameters, including the number of cells $L$, the number of users $K$, the location information of users (distance to the BS and the AoA range), the orthogonal pilot number $\tau$, etc., $W$ is obtained by combining the weight matrix calculation formula of all sides.

In Steps 3-5, the proposed GC-LI algorithm uses the weight matrix $W$ of all edges to calculate the degree $W_{\text {sum }}$ of all vertices, that is, the sum of the interference received by each user.

In Step 6, we sort the vertices from large to small according to the value of the degree $W_{\text {sum }}$ of the vertices and gets the final sorted set index of all users.

In Step 7, the algorithm first initializes the result of the final output poilt, and poilt $(:, 1)$ represents the number of all users, namely, the sorted set index of all users; poilt $(:, 2)$ represents the pilot number assigned to all users.

In Steps 8-21, the algorithm colors the $K * L$ users sequentially according to the sorted set index of all users. Steps 10-20 describe the strategy for assigning pilots to each user. The strategy is described in detail as follows. Firstly, we determine the position of pilot number $j$ in the users who have been assigned pilots through Step 11. Then, the sum interference $W_{p}(j)$ of other users who are using pilot number $j$ at this time to user $i$ is calculated through Steps 12 to 18 . Then, we find out the best pilot through Step 20, that is to say, the sum of the interference values between the user who multiplexes the pilot and the target user $i$ is the minimum, and then assign it to the user to obtain the assignment result in poilt $(i, 2)$.

Finally, all users' pilot assignment is completed in $K * L$ cycles and the pilot assignment result poilt is output.
TABLE 1: Simulation parameters.

\begin{tabular}{lc}
\hline Parameters & Value \\
\hline Number of cells $L$ & {$[1,7,19]$} \\
Cell radius $R$ & {$[300 \mathrm{~m}, 500 \mathrm{~m}, 700 \mathrm{~m}]$} \\
Number of BS antennas $M$ & {$[16,32,64,128,256,512,1024]$} \\
Number of cell users $K$ & 12 \\
Pilot length $\tau$ & 12 \\
Path loss factor $\alpha$ & 3.8 \\
SNR & $20 \mathrm{~dB}$ \\
Lognormal shadow fading & $8 \mathrm{~dB}$ \\
$\delta_{\text {shadow }}$ & $3.5 \mathrm{GHz}$ \\
Carrier frequency $f_{s}$ & $\lambda / 2$ \\
Antenna spacing $D$ & 10 \\
Angle expansion $A_{s}$ & {$[16,32,64,128,256,512,1024] * 2$} \\
DFT points & 0.05 \\
Pilot overhead coefficient $\mu_{0}$ & $30 \mathrm{dBm}$ \\
Pilot symbol transmit power & $30 \mathrm{dBm}$ \\
$P_{p}$ & \\
Data symbol transmit power & \\
$P_{u}$ & \\
\hline
\end{tabular}

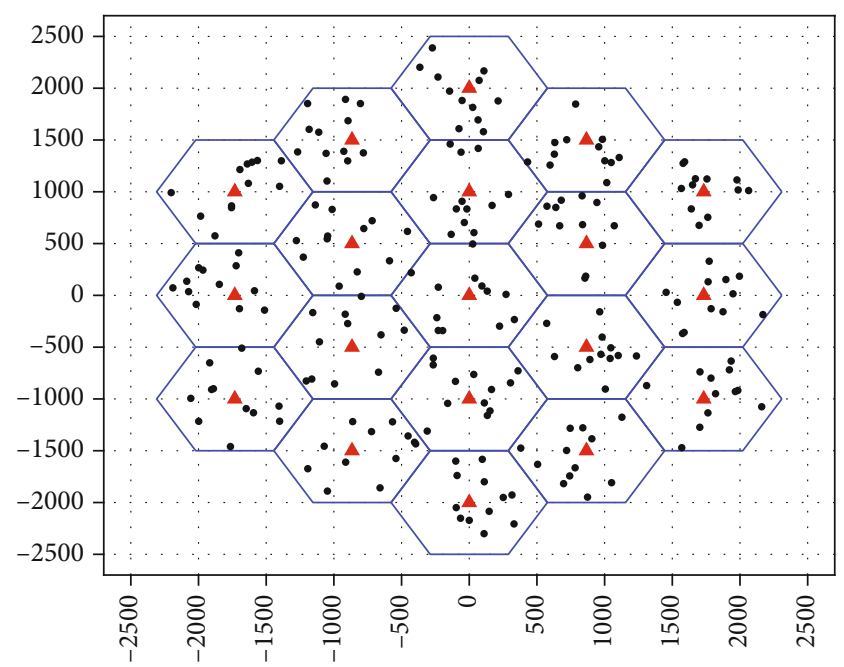

FIgURE 7: Simulation diagram of multicell multiuser random distribution.

\section{Simulation Results and Analysis}

Here, we investigate the performance of the proposed GC-LI scheme through Monte Carlo simulations. A typical hexagonal cellular network with $L$ cells is considered, where each cell has a BS with $M(M \gg K)$ antennas and $K$ single-antenna users. The users are uniformly randomly distributed in their corresponding cells in each trial. The simulation system's parameters are listed in Table 1.

Considering the antenna correlation and the mutual coupling effect, the antenna spacing $D$ is half of the carrier 


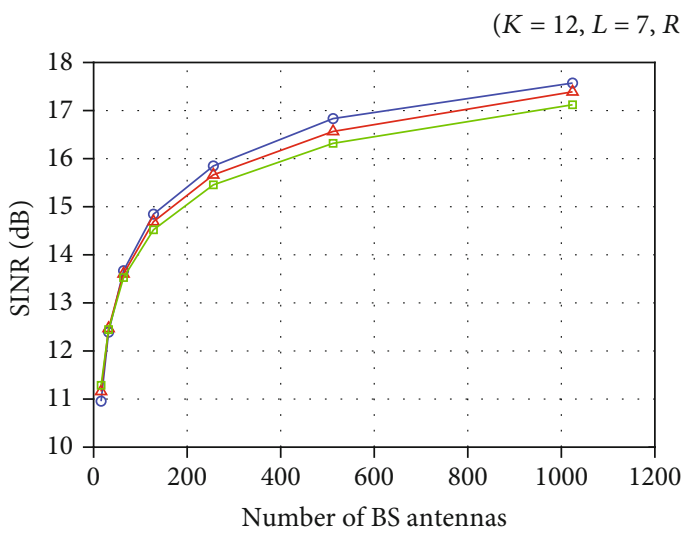

(a)

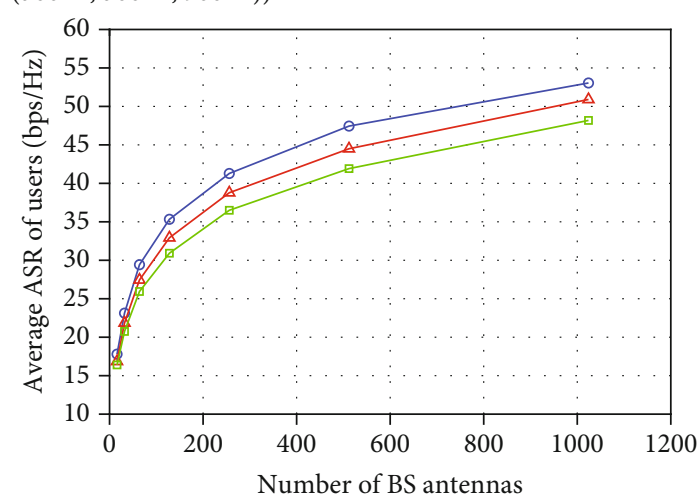

(b)

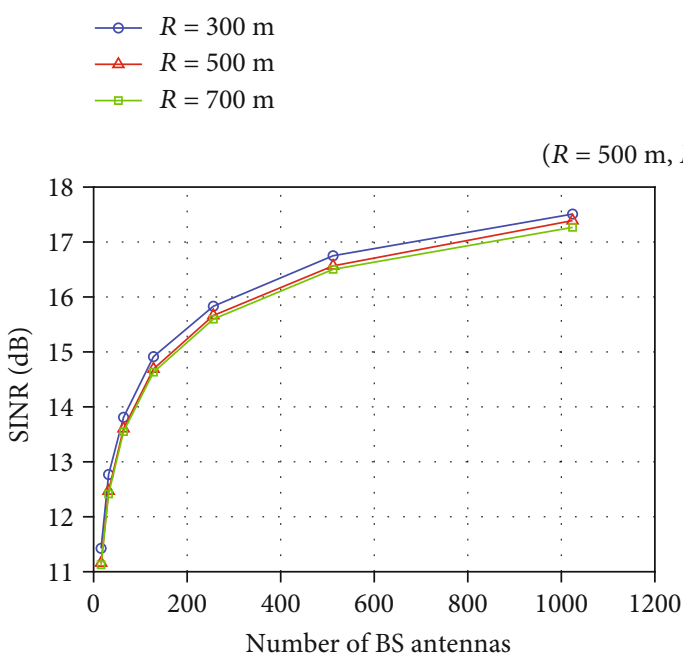

(c)

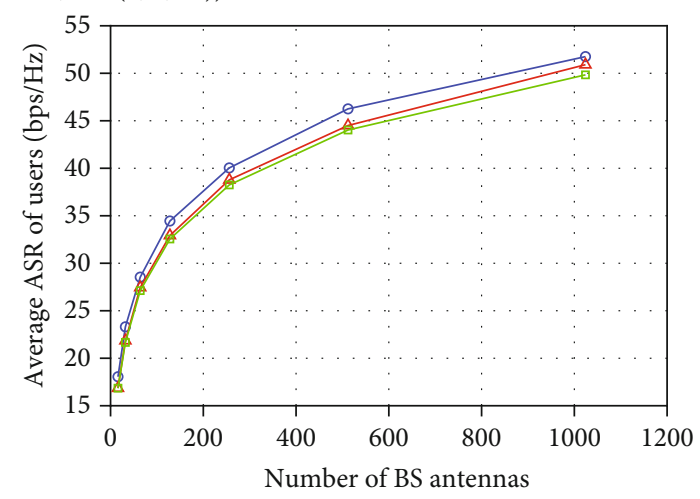

(d)

$$
\begin{aligned}
& \multimap \quad L=1 \\
& \triangle \quad L=7 \\
& \square \quad L=19
\end{aligned}
$$

FIgURE 8: Average SINR and ASR of users under various cell radii and numbers.

wavelength $\lambda$. It is assumed that all users in all cells occupy a unified time-frequency resource block, that is, users transmit signals in the cochannel simultaneously.

Figure 7 is a simulation diagram of the random distribution of multiple cells and multiple users with $L=19, K=12$, $R=500 \mathrm{~m}$. The hexagon enclosed by the blue line represents the district, the red triangle in the center of each cell represents the BS equipped with $M$ antennas, and the solid black circle represents users with a single antenna.

To verify the performance of the proposed GC-LI algorithm, we compared it with the WGC-PD algorithm in [23], the GC-PA algorithm in [24], and the VGC-PA algorithm in [31]. The WGC-PD algorithm and the GC-PA algorithm only use the large-scale fading coefficients to construct the interference graphs, which are not pinpoint enough. The VGC-PA algorithm directly combines the AoA factor and the large-scale fading coefficient to obtain the metric interference function and then constructs the interference graph. However, when the users' AoAs are nonoverlapping, we can eliminate the ICI without considering the large-scale fad- ing coefficient. The proposed GC-LI algorithm divides AOA into four kinds to discuss and then combined with the largescale fading coefficient to construct the interference graph to improve the accuracy in different situations.

With the decrease of cell radius and the increase of cell number in the 5G scenery, the effect of the traditional pilot allocation becomes worse. In this paper, we propose the GC-LI algorithm. Firstly, the influence of various radii and numbers of cells is simulated.

From Figures 8(a) and 8(b), we can see that with the increase of the radius of each cell, the average SINR and ASR of users decrease. The main reason is that the cell's radius increases so that the corresponding large-scale fading coefficient increases and the users' performance decreases slightly. The simulation results also reflect that the proposed GC-LI algorithm can perform better under various radii of cells. Even if the increase of radius leads to performance degradation, the algorithm is still applicable, reflecting the rationality of the proposed GC-LI algorithm. In Figures 8(c) and $8(\mathrm{~d})$, with the increase of the number of cells, the average 


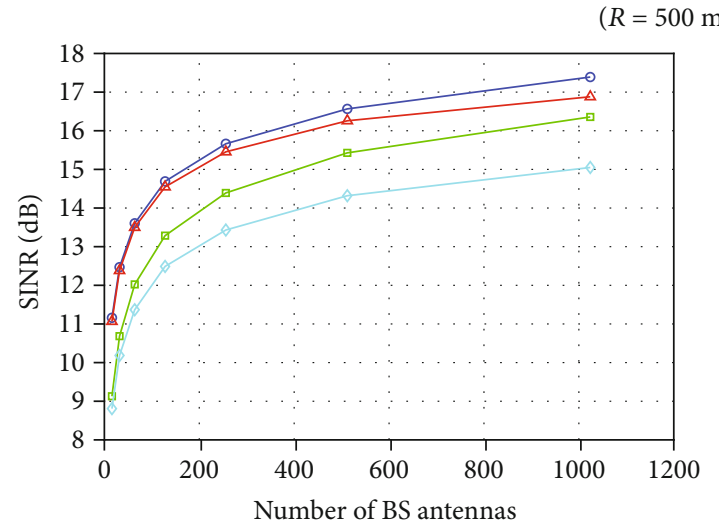

(a)

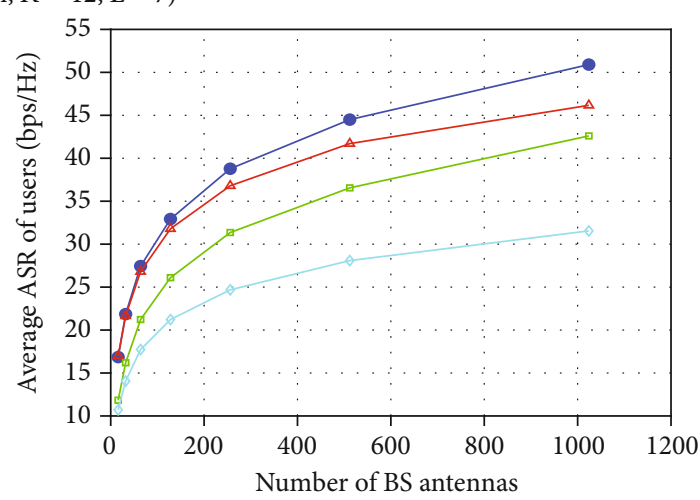

(b)

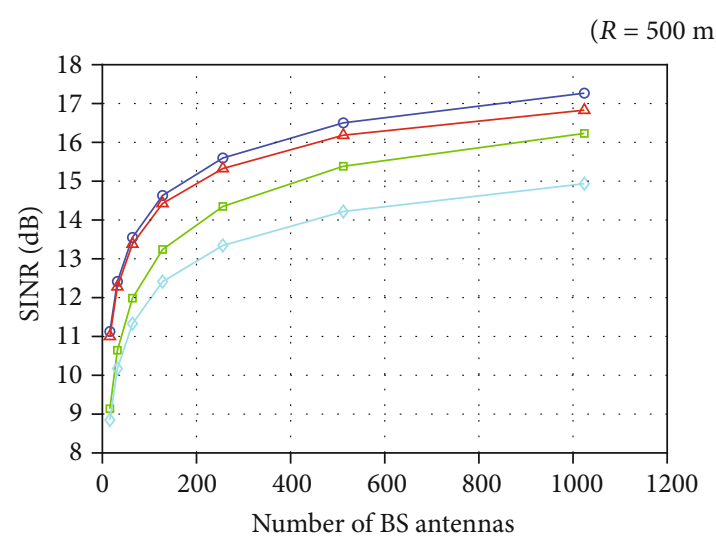

(c)

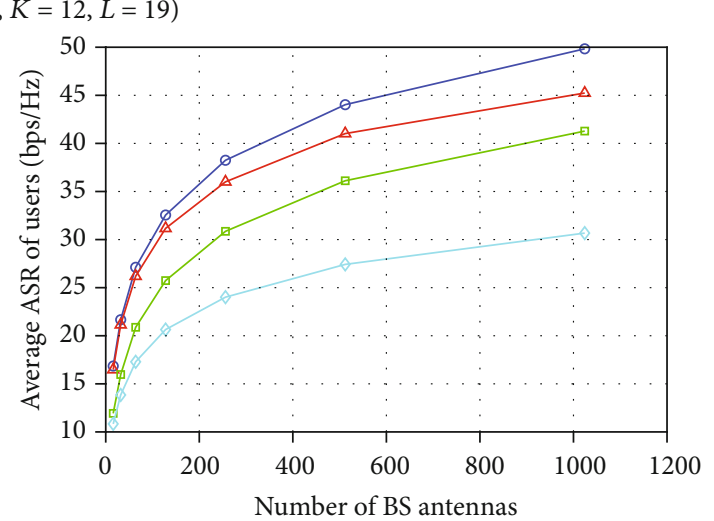

(d)

$$
\begin{array}{ll}
\multimap \text { Proposed GC-LI } & \square \text { WGC-PD } \\
\triangle \text { VGC-PA } & - \text { GC-PA }
\end{array}
$$

FIgURE 9: Average SINR and ASR of users under different pilot assignment algorithms.

SINR and ASR of users slightly decrease. When the number of orthogonal pilots is fixed, the increase of the number of users multiplexing pilot leads to the aggravation of PC. The proposed algorithm dynamically allocates the optimal pilot according to the actual interference value between users to minimize the ICI of the multiplexed pilot ad maximize the system capacity. The simulation results show that the proposed GC-LI algorithm can be applied to various radii and number cells.

Figure 9 simulates and compares the influence of the antenna number $M$ on users' average SINR and ASR of the four different pilot assignment strategies. In Figures 9(a) and $9(\mathrm{~b})$, when the number of antennas $M=1024$, the average SINR of the proposed GC-LI algorithm is about $0.5 \mathrm{~dB}$ higher than that of the VGC-PA algorithm and is $1 \mathrm{~dB}$ and $2 \mathrm{~dB}$ higher than those in the WGC-PD algorithm and GCPA algorithm, respectively. The average cell ASR of the proposed GC-LI algorithm is better than the VGC-PA algorithm about $5 \mathrm{bps} / \mathrm{Hz}$ and better than the WGC-PD algorithm and the GC-PA algorithm about $7 \mathrm{bps} / \mathrm{Hz}$ and $18 \mathrm{bps} / \mathrm{Hz}$, respectively.

To further verify the generality of the proposed pilot allocation algorithm, we change the number of cells $L$ to 19 , namely, for the target cell, there are both primary and sec- ondary peripheral cells. Through Figures 9(c) and 9(d), we can see that the performance of the proposed GC-LI algorithm is also better than the other three algorithms. We can also see that the performance of the GC-LI algorithm is always better than the comparison algorithm under different antenna numbers.

The GC-PA algorithm allocates the least used pilots. It only distributes the pilots equally, without considering the amount of ICI that directly affects performance. The WGC$\mathrm{PD}$ algorithm first finds the multiplexed user with the minimum interference with the target user and then assigns the pilot to the target user. The proposed GC-LI algorithm takes the postprocessing DFT filtering process to eliminate the interference between users with nonoverlapping AoAs. Compared with the VGC-PA algorithm, it considers the different influences of AoA on two-way users. It constructs the interference graph according to different situations, so the interference is evaluated more accurately.

Figure 10 compares the CDF of users' uplink average SINR and ASR under different pilot assignment algorithms.

We can see that compared with the three algorithms, the GC-LI algorithm proposed in this paper has a higher average SINR and ASR under the same CDF. Taking CDF $=0.5$ as an example, in Figure 10(a), the SINR of the GC-LI algorithm is 


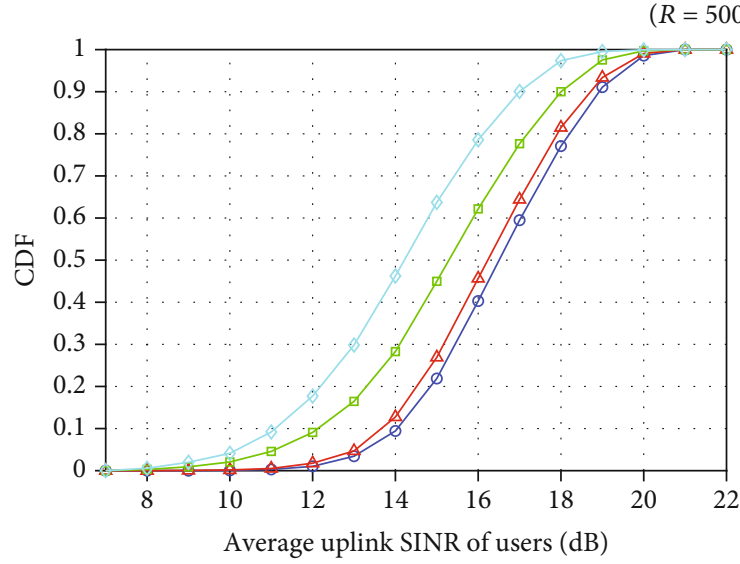

(a)

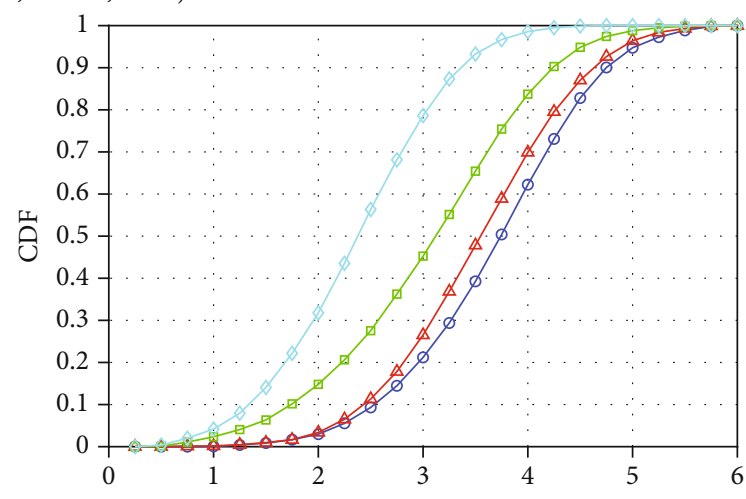

(b)

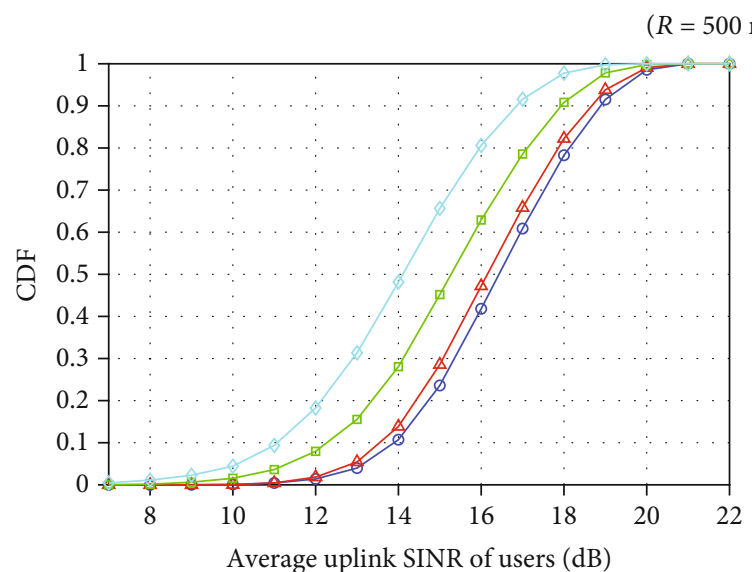

(c)

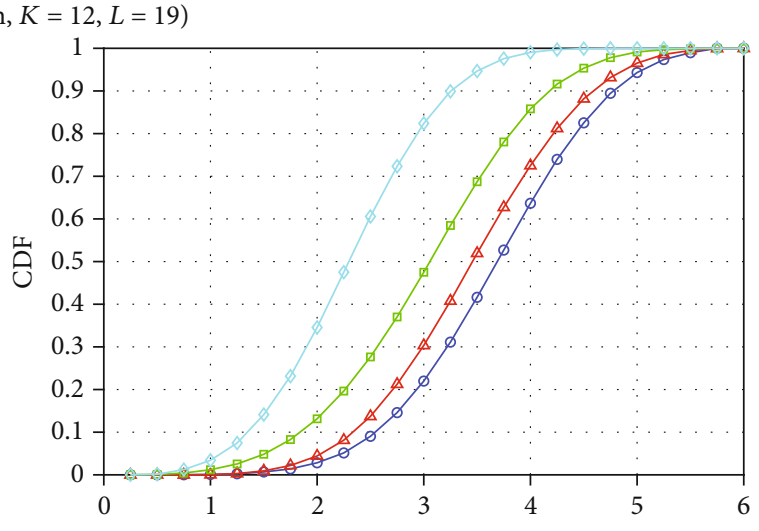

(d)

$$
\begin{aligned}
& \rightarrow \text { Proposed GC-LI } \rightarrow \text { WGC-PD } \\
& \triangle \text { VGC-PA }
\end{aligned}
$$

Figure 10: CDF of users' uplink SINR and ASR under different pilot assignment algorithms.

$16.5 \mathrm{~dB}$, by about $0.3 \mathrm{~dB}$ compared with the VGC-PA algorithm, $1.3 \mathrm{~dB}$ compared with the WGC-PD algorithm, and $2.2 \mathrm{~dB}$ compared with the GC-PA algorithm. In Figure 10(b), the ASR of the GC-LI algorithm is $3.7 \mathrm{bps} / \mathrm{Hz}$, by about $0.2 \mathrm{bps} / \mathrm{Hz}$ compared with the VGC-PA algorithm, $0.6 \mathrm{bps} / \mathrm{Hz}$ compared with the WGC-PD algorithm, and $1.5 \mathrm{bps} / \mathrm{Hz}$ compared with the GC-PA algorithm. Obviously, the overall performance of the proposed GC-LI algorithm has been greatly improved.

We further simulate the CDF of uplink average SINR and the ASR of users under the four different pilot assignment algorithms when the number of cells $L$ changes to 19 . From Figures $10(\mathrm{c})$ and $10(\mathrm{~d})$, we can see that with the change of CDF, the GC-LI algorithm has a higher average SINR and ASR compared with the comparison algorithms. It shows that the proposed GC-LI algorithm can improve system performance in multicell multiuser massive MIMO scenarios.

\section{Conclusion}

This paper researches the PC suppression technology based on pilot assignment. We propose a GC-LI algorithm, which is suitable for various radii and numbers of cells. Specifically, after the traditional channel estimation process, the spatial selection characteristics of the antenna array direction vector and the postprocessing DFT filtering process are used to eliminate the mutual interference between users with nonoverlapping AoAs. This paper also proposes a method to measure the potential ICI strength between any two users based on the correlation and distance of AoA. Considering the four situations existing between users, an ICI graph is constructed to describe the potential ICI relationship between any two users in the system. Finally, we propose the GC-LI algorithm, which uses the idea of graph coloring to assign appropriate pilots to minimize the ICI between users, thereby suppressing the PC and improving system performance. Simulation results show that users' average SINR and ASR of the proposed algorithm are improved by comparing with the three pilot assignment algorithms based on graph coloring.

\section{Data Availability}

To be frank, I derived the writing material from different journals as provided in the references. A MATLAB tool has been utilized to simulate our concept. 


\section{Conflicts of Interest}

The authors declare that there is no conflict of interest regarding the publication of this paper.

\section{Acknowledgments}

This research was funded by the National Key Research and Development Program of China under Grant No. 2018 YFB2100100.

\section{References}

[1] J. Zhang, E. Björnson, M. Matthaiou, D. W. K. Ng, H. Yang, and D. J. Love, "Prospective multiple antenna technologies for beyond 5G," IEEE Journal on Selected Areas in Communications, vol. 38, no. 8, pp. 1637-1660, 2020.

[2] S. A. Busari, K. M. S. Huq, S. Mumtaz, L. Dai, and J. Rodriguez, "Millimeter-wave massive MIMO communication for future wireless systems: a survey," IEEE Commun. Surveys \& Tutorials, vol. 20, no. 2, pp. 836-869, 2017.

[3] W. Yuan, X. Yang, and R. Xu, "A novel pilot decontamination scheme for uplink massive MIMO systems," Procedia Computer Science, vol. 131, pp. 72-79, 2018.

[4] S. Ghosh, D. De, and P. Deb, "Energy and spectrum optimization for $5 \mathrm{G}$ massive mimo cognitive femtocell based mobile network using auction game theory," Wireless Personal Communications, vol. 106, no. 2, pp. 555-576, 2019.

[5] A. A. Abdulateef, S. M. Ibrahim, A. H. Mohammed, and I. A. Abdulateef, "Performance analyses of channel estimation and precoding for massive MIMO downlink in the TDD system," in 2020 4th International Symposium on Multidisciplinary Studies and Innovative Technol. (ISMSIT), pp. 1-6, Istanbul, Turkey, November 2020.

[6] Z. Gong, C. Li, and F. Jiang, "Pilot decontamination in noncooperative massive MIMO cellular networks based on spatial filtering," IEEE Transactions on Wireless Communications, vol. 18, no. 2, pp. 1419-1433, 2019.

[7] T. Kim, K. Min, M. Jung, and S. Choi, "Scaling laws of optimal training lengths for TDD massive MIMO systems," IEEE Transactions on Vehicular Technology, vol. 67, no. 8, pp. 7128-7142, 2018.

[8] X. Ge, Y. Sun, H. Gharavi, and J. Thompson, "Joint optimization of computation and communication power in multi-user massive MIMO systems," IEEE Transactions on Wireless Communications, vol. 17, no. 6, pp. 4051-4063, 2018.

[9] A. Sheikhi, S. M. Razavizadeh, and I. Lee, "A comparison of TDD and FDD massive MIMO systems against smart jamming," IEEE Access, vol. 8, pp. 72068-72077, 2020.

[10] L. Ge, Y. Zhang, G. Chen, and J. Tong, "Compression-based lmmse channel estimation with adaptive sparsity for massive MIMO in 5G systems," IEEE Systems Journal, vol. 13, no. 4, pp. 3847-3857, 2019.

[11] I. Khan and D. Singh, "Efficient compressive sensing based sparse channel estimation for 5G massive MIMO systems," AEU-International Journal of Electron. and Commun., vol. 89, pp. 181-190, 2018.

[12] Y. Han, S. Jin, C. K. Wen, and X. Ma, "Channel estimation for extremely large-scale massive MIMO systems," IEEE Wireless Communications Letters, vol. 9, no. 5, pp. 633-637, 2020.
[13] M. Feng and Y. Xu, "Low-complexity linear precoding for pilot contamination mitigation in multi-cell massive mimo systems," in 2017 International Symposium on Intelligent Signal Processing and Communication Systems (ISPACS), pp. 807811, Xiamen, China, Novemeber 2017.

[14] R. Mai, T. le-Ngoc, and D. H. Nguyen, "Two-timescale hybrid RF-baseband precoding with MMSE-VP for multiuser massive MIMO broadcast channels," IEEE Transactions on Wireless Communications, vol. 17, no. 7, pp. 4462-4476, 2018.

[15] A. Almamori and S. Mohan, "A spectrally efficient algorithm for massive MIMO for mitigating pilot contamination," in 2017 IEEE 38th Sarnoff Symposium, pp. 1-5, September 2017.

[16] T. K. Nguyen, H. H. Nguyen, and T. H. Nguyen, "Multiuser massive MIMO systems with time-offset pilots and successive interference cancellation," IEEE Access, vol. 7, pp. 132748132762, 2019.

[17] F. Yang, P. Cai, H. Qian, and X. Luo, "Pilot contamination in massive MIMO induced by timing and frequency errors," IEEE Transactions on Wireless Communications, vol. 17, no. 7, pp. 4477-4492, 2018.

[18] T. C. Mai, H. Q. Ngo, M. Egan, and T. Q. Duong, "Pilot power control for cell-free massive MIMO," IEEE Transactions on Vehicular Technology, vol. 67, no. 11, pp. 11264-11268, 2018.

[19] T. Van Chien, E. Bjornson, and E. G. Larsson, "Joint pilot design and uplink power allocation in multi-cell massive MIMO systems," IEEE Transactions on Wireless Communications, vol. 17, no. 3, pp. 2000-2015, 2018.

[20] T. A. Le, T. Van Chien, M. R. Nakhai, and T. Le-Ngoc, "Pareto-optimal pilot design for cellular massive MIMO systems," IEEE Transactions on Vehicular Technology, vol. 69, no. 11, pp. 13206-13215, 2020.

[21] Y. Wu, T. Liu, M. Cao, L. Li, and W. Xu, "Pilot contamination reduction in massive MIMO systems based on pilot scheduling," EURASIP Journal on Wireless Commun. and Netw., vol. 2018, no. 1, pp. 1-9, 2018.

[22] N. Akbar, S. Yan, N. Yang, and J. Yuan, "Location-aware pilot allocation in multicell multiuser massive MIMO networks," IEEE Transactions on Vehicular Technology, vol. 67, no. 8, pp. 7774-7778, 2018.

[23] X. Zhu, L. Dai, Z. Wang, and X. Wang, "Weighted-graph-coloring-based pilot decontamination for multicell massive MIMO systems," IEEE Transactions on Vehicular Technology, vol. 66, no. 3, pp. 2829-2834, 2017.

[24] X. Zhu, L. Dai, and Z. Wang, "Graph coloring based pilot allocation to mitigate pilot contamination for multi-cell massive MIMO systems," IEEE Communications Letters, vol. 19, no. 10, pp. 1842-1845, 2015.

[25] J. Xu, P. Zhu, J. Li, and X. You, "Deep learning-based pilot design for multi-user distributed massive MIMO systems," IEEE Wireless Communications Letters, vol. 8, no. 4, pp. 1016-1019, 2019.

[26] K. Kim, J. Lee, and J. Choi, "Deep learning based pilot allocation scheme (DL-PAS) for 5G massive MIMO system," IEEE Communications Letters, vol. 22, no. 4, pp. 828-831, 2018.

[27] R. Khan, P. Kumar, D. N. K. Jayakody, and M. Liyanage, "A survey on security and privacy of $5 \mathrm{G}$ technologies: potential solutions, recent advancements, and future directions," IEEE Communications Surveys \& Tutorials, vol. 22, no. 1, pp. 196$248,2020$. 
[28] X. Nie and F. Zhao, "Joint pilot allocation and pilot sequence optimization in massive MIMO systems," IEEE Access, vol. 8, pp. 60637-60644, 2020.

[29] Y. Kabalci, "5G mobile communication systems: fundamentals, challenges, and key technologies," in Smart Grids and Their Communication Systems. Energy Systems in Electrical Engineering, pp. 329-359, Springer, 2019.

[30] H. Yin, D. Gesbert, M. Filippou, and Y. Liu, "A coordinated approach to channel estimation in large-scale multipleantenna systems," IEEE Journal on Selected Areas in Communications, vol. 31, no. 2, pp. 264-273, 2013.

[31] H. T. Dao and S. Kim, "Vertex graph-coloring-based pilot assignment with location-based channel estimation for massive MIMO systems," IEEE Access, vol. 6, pp. 4599-4607, 2018. 